\title{
Development of a corporate information system for companies
}

\author{
Olesya Golubeva ${ }^{1 *}$ and Alina Pogorelova ${ }^{1}$ \\ ${ }^{1}$ Don State Technical University, Gagarin sqr, 1, Rostov-on-Don, 344003, Russia
}

\begin{abstract}
Modern trends in the creation and development of corporate information systems (CIS) are largely determined by their consumer quality. A model of building a corporate information system (CIS) of a universal open architecture is presented. The possibility of using CIS for managing the production processes of a shoe organization is demonstrated.
\end{abstract}

\section{Corporate information systems}

Any activity of the enterprise can be represented as a set of projects, the implementation of which leads to the production of products or the achievement of the necessary results. In the current conditions of strong competition of manufacturers, the main problems in the implementation of projects at large enterprises are: ensuring control over the progress of the project within strict external conditions and deadlines, as well as organizing optimal interaction of a large number of project participants. The solution of these problems can be most effectively implemented using modern means of communication and appropriate software. At present, large organizations and administrative management structures, especially geographically distributed ones, are aware of the need to improve management and production processes. The problem of effective project management can be solved by implementing a corporate information system (CIS) in the organization, which should cover all areas of the organization's activities and combine them into a single information space. This article presents a model for building a universal open architecture CIS based on the concept of a content-oriented data warehouse.

The analysis highlights such characteristics of the quality of the CIS as availability, which implies the time of the actual operation of the system applications; manageability, which characterizes the degree of management of distributed applications; performance, defined as transaction throughput and resource use of the information system; reliability, which implies everything related to the smooth operation of applications; scalability, which provides the ability to add resources to the application in order to adequately increase the capabilities of its service; application and data security; the size of objects (text, graphics, video, audio) received by the browser from the Web server; the number of users, which can reach hundreds of thousands or millions [1].

The consumer quality of a CIS built on the basis of Web technologies is determined by the content, design, performance level, functionality, performance and security level. For the

* Corresponding author: 1354565@mail.ru 
end user, the main characteristics of the quality of the CIS are performance (low response time) and reliability (zero number of access failures).

Reduced system performance can be the result of insufficient bandwidth at peak loads, heavy load or its jumps to the servers, the transfer of dynamically changing information, the lack of connections between application servers and database servers, failures of Web services provided by third parties, the transfer of multimedia information.

When analyzing the performance of the CIS, it is necessary to take into account the system infrastructure, site content, server software and hardware, application characteristics, and network bandwidth.

The performance characteristics (performance) of an information system are becoming critical for business, and therefore their analysis and forecasting is an important task of designing and maintaining a CIS.

Server performance depends on the number and speed of processors, the amount of RAM, the bandwidth and memory capacity of the disk subsystem, and the bandwidth of the network adapter.

The operating system of a Web server largely determines such characteristics as reliability, operational efficiency, extensibility, and stability.

The content of the Website includes HTML documents, images, video and audio objects. The performance of the information system is affected by the volume and structure of the content.

The most common performance indicators of Web systems are: response time for end-toend data transmission, site response time, throughput (requests / s or Mbit / s), number of errors per second, number of visitors per day.

In general, the CIS model can be represented in the following form: $\{\mathrm{S}, \mathrm{SW}, \mathrm{HW}, \mathrm{A}, \mathrm{C}$, $\mathrm{PN}$ \}, where the characteristics of S -the infrastructure of the Web system; SW-software; HW - hardware; A - applications; C - the content of the Web site; PN - the communication network are presented.

To analyze the performance of the CIS, it is necessary to estimate the execution time of the most critical business transactions for it. Web transactions require browser, network, and server resources and implement DNS domain name service lookup processes to convert a URL to an IP address, establish a TCP connection, transmit information (request and content) over the network, and process the request on the Web server.

Conceptually, the Web system model can be represented as a closed queuing network.

The corporate information system should provide solutions to the following problems:

1. organization of a single information space for structural units directly performing work on the project;

2. storage and secure access to electronic project documentation;

3. corporate work planning;

4. management of the work execution process (continuous monitoring with the possibility of development in the field of analysis and decision support);

5. integration of the tasks of the support units into the overall work process;

6. efficient use of the organization's resources and cost optimization in the implementation of projects.

The traditional approach involves the use of a set of separate highly specialized software tools to implement the above tasks. It should be noted that this approach leads to problems of interaction of individual software products, as a rule, from different development companies and, in general, greatly reduces the effect of the use of information technologies in the organization.

The main functions of corporate information systems are shown in Figure 1. 


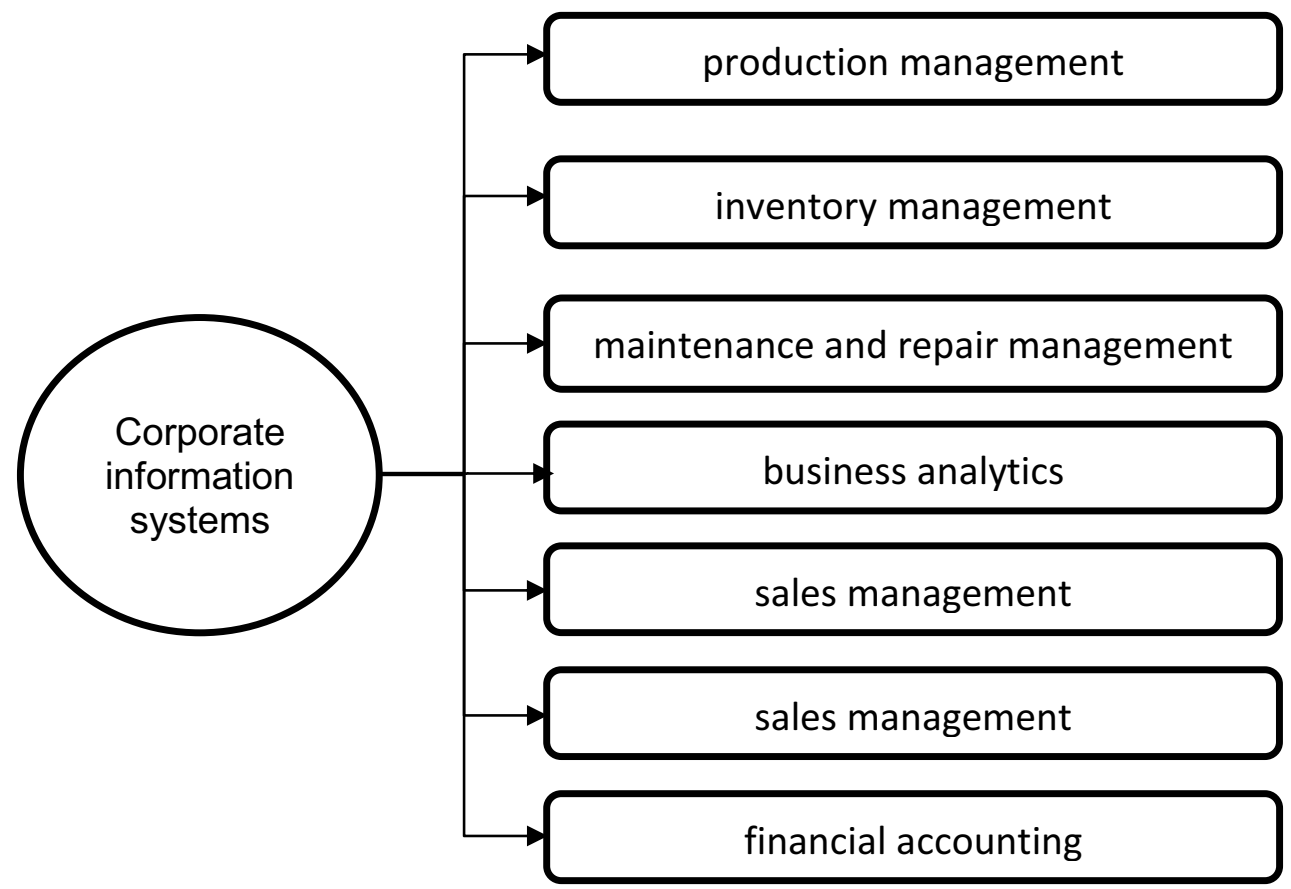

Fig. 1. Corporate information systems.

There are the following classes of software (software) designed to solve the problems of organizing the production process as a whole:

1. ERP-Enterprise Resource Planning (a set of integrated systems that allow you to create a single information space for automating the planning, accounting, control and analysis of all major business operations on enterprise resources: production, supply, storage, finance, sales (sales), etc.) [1;2];

2. CRM-Customer Relationships Management [2];

3. PLM-Product Lifecycle Management (product lifecycle management system) [3].

Within the framework of the above approaches, the solution to all the problems of the organization is declared. However, practice shows a certain narrow focus for each information system of this type. A more general approach is required to implement all the tasks of the organization. The disadvantage of existing systems is also the extremely high cost of preparing the infrastructure, the initial purchase of software, its implementation and maintenance.

In this article, we consider a model for building a CIS based on the following principles:

1) universality of the data model - the data model allows you to store arbitrary information in a semantically related form;

2) the ability to manage system metadata without programming - the proposed model provides the ability to develop and configure the system without knowledge of programming languages;

3) availability of an access rights management system - the model contains authorization and authentication mechanisms using external corporate access centers (OpenLDAP, eDirectory, ActiveDirectory);

4) high scalability of the solution-the model can operate on a wide range of software and hardware platforms, from the most common systems based on MS Windows and Intel up to high-performance and fault-tolerant clusters based on SUN Solaris and UltraSPARC 25K or IBM AIX and PowerX Cell; 
5) flexibility of hardware and software - depending on the needs of the organization, you can use the appropriate set of software and hardware, providing the ability to build disasterresistant solutions and meet the requirements of GOST for information protection;

6) use of open standards - when implementing software in accordance with this model, only open standards (SQL, JSP, XML, AJAX, HTML) are used, which are widely used;

7) using free platforms-the solution infrastructure can be built on the basis of free software, while the Linux OS family can be used as an operating system (OS), Oracle XE [4] or PostgreSQL [5] as a database management system (DBMS), and any J2EE - compliant JV can be used as an application server [6].

One of the key problems of building a CIS is to ensure information security and its compliance with the standards of the Russian Federation in this area. The CIS model presented in this article assumes the implementation of the following basic principles of information security:

1. authorization and authentication of users via an LDAP server;

2. using the HTTPS protocol to encrypt web traffic;

3. verifying md5 checksums for documents (or using a certified digital signature);

4. The access rights management system (DBMS) is integrated with the organization's staffing table, access to information is provided on the basis of a hierarchy;

5. special technology of access to DBMS objects, excluding direct access to data (bypassing the COURT);

6. the system stores a complete history of data changes;

7. access rights define the data representation;

8. the possibility of arbitrary grouping into access groups.

The organization of metadata allows you to implement the classic schemes of relations between CIS documents - one-to-one, one-to-many, many-to-many - in accordance with the theory of relational databases.

The basic object of the data storage system is a document of any structure. The document structure consists of an arbitrary set of attributes and is shown in Figure 2.

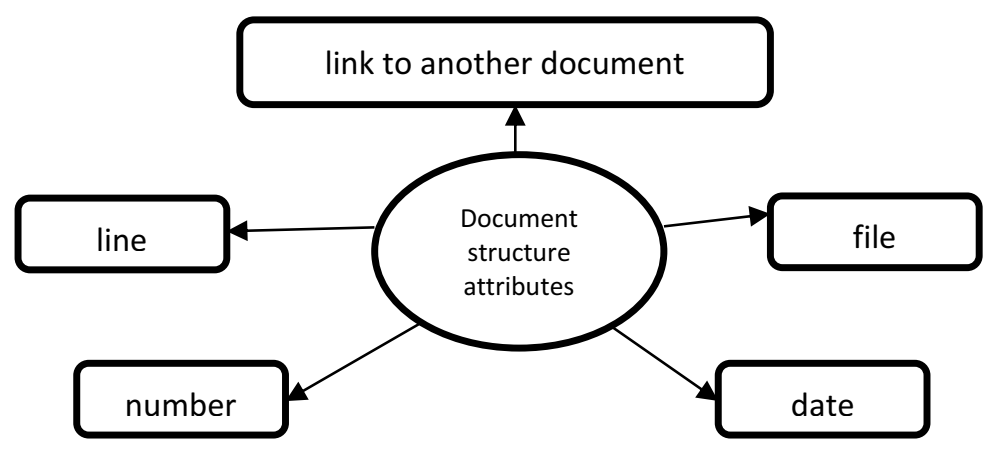

Fig. 2. The structure of the document.

This organization of information storage allows you to implement a universal mechanism for searching for information on all attributes (documents) and even on the contents of files in the case of using a DBMS that has a full-text search function.

Under the object of the CIS, we will understand an arbitrary set of documents CIS, united by the relationships described in the metadata. The object of the CIS can be a simple memo, information about an employee of the organization, the content of the entire project, etc.

The proposed model allows you to implement a project approach to all the activities of the organization. It allows you to manage a portfolio of projects from the level of strategic 
planning of the organization's development to the level of detail of specific works, their performers, deadlines, and other resources. This can be either a project for the construction of the Baltic Pipeline system, or a project for the implementation of the educational process at the department or at the university as a whole.

To implement the process approach to the implementation of project work in the CIS, it is also necessary to use the work flow management subsystem (WorkFlow), which includes the following functions:

1. setting routes for passing CIS objects without programming;

2. existence of typical WorkFlow elements-approval, approval, notification;

3 . using route templates and documents.

The connecting link of GIS is the subsystem of electronic document management, which combines both the functions of external correspondence of the organization and internal document management. This allows you to trace the path of any document from its creation by the contractor to the approval of the management and sending to external organizations with control of the passage at each stage.

\section{Developed corporate information system for shoe companies}

Information support has been developed, including a set of information necessary for the implementation of computer-aided design and control of shoe production. This is a set of OBUVPRO databases containing lists of technological equipment: materials, equipment, devices, tools, names of operations, time standards, material consumption standards, conditions for selecting operations and equipment for computer-aided design of technical equipment, reference books for coding product design elements and their parameters, etc.

The software system "Obuvpro+" has been developed, which provides the industry enterprises with a transition to a qualitatively new level of solving design and technological problems and obtaining economic results.

The database "Automated design of technological processes of assembly of footwear "Obuvpro+" consists of the developed "Integrated software system for shoe enterprises", designed to improve the quality of work of shoe enterprises.

There are several platforms that fully cover the task of building information systems. Among them, the Oracle platform is selected. It is characterized by high scalability from free solutions to enterprise solutions; full multi-platform and the highest degree of protection. The system is created within the framework of a multi-platform architecture and consists of the following subsystems: a Web application, a Database Management System (DBMS), and a system for generating analytical reports.

The web application is a point of access for authorized persons to the functions and data of the system. The web-application provides: access to the information base of the company's employees, when editing data, it is possible to use fields that are required to be filled in by the user, the ability to receive tips on filling in fields, the ability to select field values from the directory that is filled in in administration mode, the ability to save navigation through the portal when its graphical elements are disabled.

The database management system provides: storage of all data, isolation by the level of data reading, support for transactions and consistent state of the database at any time.

The software system is based on the principles of an architecture focused on providing access to software components and data through a web interface and providing a single way to access the system through both local and global networks. The system software allows users to work with it using the technology of "thin" clients through a web browser on the Internet network. Data access is protected by password authentication of users, monitoring of user actions, and the ability to roll back the result of illegal user actions. Unauthorized users are not allowed to access the system. 


\section{Conclusion}

In conclusion, we note that this article describes the principles of building a universal model of a corporate information system with the ability to configure it for specific application tasks. The CIS implemented on the basis of this model can be used in various fields of activity.

A distinctive feature of this model is a unified approach to storing and processing all the data of the organization, regardless of the information consumers, and then presenting the information in the form necessary for each consumer. In the implemented model, it is possible to forward complex structured CIS objects with the required level of detail to any consumer, taking into account access rights. The described approach to the design of CIS provides high reliability and security of information storage.

\section{References}

1. K. Brueckner, J. Heidnefelder, S. Odenwald, T.L. Milani, Footwear Science, 3(S1), 1819 (2011)

2. V.N. Kraev, Management Decision Making Methods (2014)

3. J.C. Choi, C. Kim, J. Mater. Process Technol., 110 (2013)

4. G. Colombo, D. Ferretti, U. Cugini, Proceedings of international symposium on advanced geometric modelling for engineering applications, 2-15 (2014)

5. G. La Rocca, L. Krakers, M.J.L. van Tooren, Proceedings 9th symposium on multidisciplinary analysis and optimization, 2-13 (2014)

6. A. Luximon, Handbook of Footwear Design and Manufacture, 64, 5, 416 (2013)

7. A. Healy, D. Dunning, N. Chockalingam, Footwear Science, 3(S1), 69-70 (2011)

8. T. Nishiwaki, J. Tateishi, (2011), Footwear Science, 3(S1), 122-123 (2011)

9. Y.M. Tang, K.C. Hui, Computer-Aided Design, 43, 1841-1848 (2011)

10. L. A. Ginis, Statistical Methods of Quality Control and Management, Applied Software (2019)

11. A.K. Ershov, Quality Management (2017)

12. S.I. Solonin, Control Charts Methods (2014)

13. I.V. Maruseva, Modern Management, Classic and Applied Aspects (2018) 